\title{
Estimating thermal transmittance of the aluminium window profiles in practice
}

\author{
Arkadiusz Witek $^{*}, 1$, and Barbara Pietruszka ${ }^{1}$ \\ ${ }^{1}$ Thermal Physics, Acoustics and Environment Department, Building Research Institute, Ksawerów 21, 02-656 Warsaw, Poland
}

\begin{abstract}
Calculation of the heat flow through the air cavities in the EN ISO 10077-2:2017 standard for the determination of the thermal transmittance of window profiles uses models based on the equivalent thermal conductivity method. The method takes into account the radiative heat exchange in a simplified or accurate manner. In the first case, the heat exchange depends on the average temperature in cavity, in the second case - it is determined accurately by the ray tracing method. It is also of importance to differentiate emissivity of surfaces due to aging or painting what influences calculation time. In this work, the impact of the calculation method and the impact of simplifications in modelling ot the untreated surfaces on the value of the thermal transmittance of aluminium profiles was analysed on the example of a real series of products. Comparing the simplified and accurate method of determining the radiation exchange in cavities, the differences in the thermal transmittances of window profiles were up to $22 \%$. The differences between the most simplified and the most accurate modelling of the surfaces emissivity reached $23 \%$.
\end{abstract}

\section{Introduction}

Aluminium profiles are commonly used in the production of windows, doors and facades. They are characterized by the highest heat transmittance in comparison with profiles made of PVC or wood. The impact of the aluminium profiles on the final value of the heat transmittance of a window is significant, therefore it is important to determine the thermal characteristics of these profiles as precisely as possible.

Often some surfaces of considered profiles are subjected to painting, which causes a change in their emissivity. In some cases also deterioration of emissivity occurs as a result of aging of the element (aluminium oxidation process). Taking into account the diversity of the surface properties of aluminium for the profiles of complex geometry significantly increases the calculation time.

Experimental and numerical validation (by means of computational fluid mechanics) of EN ISO 100772:2017 standard simplified and accurate methods, [1], have been the subject of a number of research works. In [2], the thermal transmittance, $U_{f}$, of the aluminum profile obtained using the simplified method of the standard was more favourable by $4-6 \%$ than the results of experimental tests in the hotbox. Taking into account measurement uncertainties, the difference becomes even smaller. It was also shown that a change of the emissive properties of surface can cause a thermal transmittance difference up to $18 \%$. Comparison of the thermal transmittance obtained by calculations with the results of experimental tests on the one aluminium profile and one PVC profile shows that more adequate results can be obtained using the accurate method, [3]. The influence of the transformation of irregularly shaped voids into rectangular voids on the accuracy of the prediction of heat transfer by convection and conduction was analysed in [4]. The conclusion was that simplifications can lead to significant differences in the assessment of heat flux.

The above review shows that the calculation method and the value of emisivity coefficient can be a source of considerable differences in predicting thermal transmittance, so the main goals of this work were (i) to determine the differences between the predictions of the thermal transmittance by the EN ISO 10077-2:2017 standard simplified and accurate method and (ii) to determine the differences between the predictions of models with different accuracy in mapping the real lowemissivity surfaces into the numerical model. Numerical tests were also carried out to illustrate the radiative heat exchange in rectangular and trapezoidal cavities with the same and varied emissivities of cavity sides. Based on the results of the performed calculations also the impact of profile construction on its thermal transmittance was analysed, taking into account different variants of profile insulation.

\section{Mathematical models}

Determining the thermal transmittance of profile by PNEN ISO 10077-2:2017 standard, [1], is based on using programs for solving the partial differential equation of the steady-state heat equation. The most demanding part of the procedure is predicting the heat flow in the air cavities where the conduction, convection and radiation take place. Two methods are proposed in ISO 10077-2: 2017: the simplified method and the accurate method. Brief description of this methods will be given below.

\footnotetext{
* Corresponding author: a.witek@,itb.pl
} 
The first method is called the single equivalent thermal conductivity method, second - the radiosity method, although it also takes into account convection and conduction.

Equivalent thermal conductivity used for the calculation of heat flow through the air cavities as a consequence of the convection and conduction, $\lambda_{e q, c c}$, is determined form the equation, [1]:

$\lambda_{\text {eq,cc }}=\lambda_{\text {air }} \cdot N u=\lambda_{\text {air }} \cdot\left\{\begin{array}{c}1 \text { if } b<5 \mathrm{~mm} \\ \text { else } \max \left(1, \frac{d \cdot C \cdot \Delta T^{1 / 3}}{\lambda_{\text {air }}}\right)\end{array}\right.$,

where $N u$ is Nusselt number, $\lambda_{\text {air }}$ - conductivity of still air, $b, d$ - width and depth of the rectangular air cavity, $\Delta T$ - maximum surface temperature difference in the cavity, $C$ - constant equal to $0.73 \mathrm{~W} /\left(\mathrm{m}^{2} \cdot \mathrm{K}^{4 / 3}\right)$.

In order to determine conductivity $\lambda_{\text {eq, cc }}$ in the case of the air cavities with non-rectangular shapes, the outline of the cavity is replaced by an equivalent rectangular outline. Section under the rectangular outline should have the same surface area $\left(A=A^{\prime}\right)$ and the same aspect ratio $\left(d / b=d^{\prime} / b^{\prime}\right)$ as the original one, Fig. 1 . In the radiosity method, the equivalent rectangular section is also rotated so that its sides are in accordance with the direction of heat flow. In the single equivalent thermal conductivity method, the sides of the equivalent rectangular section remain parallel to the main sides of the original outline.

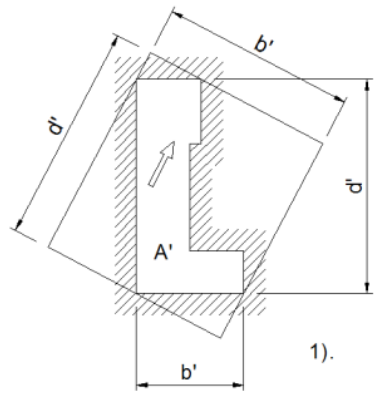

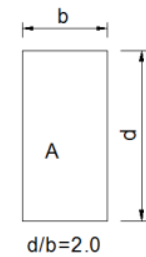

2).

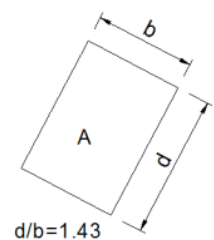

3).
Fig. 1. Methods of creating the equivalent rectangular section from the initial outline (1) in the simplified (2) and accurate method (3). The arrow indicates the direction of heat flow.

Heat exchange by radiation in the radiosity method assumes that the elementary surfaces that come from the division of the boundaries surrounding the air voids are isothermal, opaque, of uniform emissivity and behave like grey bodies. In the radiosity model of standard [1] each elementary surface $i$ is represented by the so called adjacent node and the black surface node. The heat flux between two black surface nodes $i$ and $j$ is defined as, [1]:

$$
Q_{i j}=\sigma \cdot A_{i} \cdot F_{i j} \cdot\left(T_{s b, i}^{4}-T_{s b, j}{ }^{4}\right),
$$

where $\sigma$ is the Stefan-Boltzman constant equal to $5.67 \cdot 10^{-8} \mathrm{~W} /\left(\mathrm{m}^{2} \cdot \mathrm{K}^{4}\right), A_{i}-$ the area of the elementary surface, $F_{i j}$ - view factor from surface $i$ to surface $j$, $T_{s b, i}, T_{s b, j}$ - the absolute temperatures of black surface node $i$ and $j$. The heat flow between the black surface node and adjacent node of surface $i$ is expressed by the formula, [1]:

$$
Q_{i}=\sigma \cdot A_{i} \frac{\varepsilon_{i}}{1-\varepsilon_{i}}\left(T_{s b, i}^{4}-T_{s, i}^{4}\right),
$$

where $\varepsilon_{i}$ is the total hemispherical emissivity of surface $i, T_{s, i}$ - the absolute temperature of adjacent node of the elementary surface $i$.

In the simplified method, heat transfer by radiation is included in the equivalent conductivity of air cavity accordingly to the following expression, [1]:

$$
\lambda_{e q, c c r}=d \cdot\left(h_{c}+h_{r}\right)=\lambda_{e q, c c}+d \cdot h_{r},
$$

where $h_{c}$ and $h_{r}$ are the convective and radiative heat transfer coefficients. The radiative one is calculated from formula, [1]:

$$
h_{r}=4 \sigma \cdot T_{m}{ }^{3} \cdot E \cdot F,
$$

where $E-$ is the intersurface emittance equal to $\left(1 / \varepsilon_{1}+1 / \varepsilon_{2}-1\right)^{-1}, \varepsilon_{1}, \varepsilon_{2}-$ the emissivities of the surfaces perpendicular to heat flow, $F$ - the view factor for a rectangular section equal to $1 / 2\left(1+\sqrt{1+(d / b)^{2}}-d / b\right), \quad T_{m}-$ mean from maximum and minimum temperature of surfaces surrounding the air cavity.

Summarizing, the basic differences between the accurate and simplified method are the way of determining heat transfer by radiation and the method of adopting an equivalent rectangular section. The phenomena of heat conduction through air and heat transfer by convection are described by the same formula. So, the term 'accurate method' in real refers mostly to radiative part of the methods. A more detailed description of the methods is available in [1].

\section{Calculations}

\subsection{Input data}

In this work, six different calculation models were analysed on the series of 27 different profiles in 3 thermal variants (insulation material was placed in different configurations into the air cavities). Specific feature for this series of products is the presence of air cavities with non-parallel (sloping) sides. Cross-section of an example profile from the analysed series is given in Fig. 2. Profiles are available in three thermal variants: 'full', 'plus' and 'standard'. In the 'full' version, 2 types of insulation made of foamed materials have been added compering to 'standard' version: one in the cavity with the glass panel (element No. 6 in Fig. 2) and one in the cavity between thermal breaks (element No. 7 in Fig. 2). In the 'plus' variant there is only insulation between thermal breaks, while in the 'standard' version there is no insulation in any of the cavity. Even in the 'plus' and 'full' variants, not all air cavities between thermal breaks were filled with insulation due to the insufficient width of some cavities (element No. 5 in Fig. 2). Detailed data on the number of insulated/non-insulated cavities are given in Tab. 1. Profiles were divided into 4 groups: 
'casement' - profiles with openable leaf, 'fixed' - there is no openable part in profile, 'fixed, with narrow cavities' - fixed profile with not all cavities between thermal breaks filled with the insulation because of presence of too narrow cavities, 'corner' - the corner window profiles.

Heat conductivity of the materials of the profile components were as follows: aluminium $-160 \mathrm{~W}(\mathrm{~m} \cdot \mathrm{K})$, polyamide $-0.3 \mathrm{~W}(\mathrm{~m} \cdot \mathrm{K}), \mathrm{EPDM}-0.25 \mathrm{~W}(\mathrm{~m} \cdot \mathrm{K})$, panel insulation $-0.035 \mathrm{~W}(\mathrm{~m} \cdot \mathrm{K})$, insulation near glass unit $0.038 \mathrm{~W}(\mathrm{~m} \cdot \mathrm{K})$, insulation between thermal breaks $0.031 \mathrm{~W}(\mathrm{~m} \cdot \mathrm{K})$, (see also Fig. 2). The following boundary conditions were assumed on the side of conditioned rooms: air temperature $T_{i}=20^{\circ} \mathrm{C}$, heat transfer coefficient $h_{i}=7.7 \mathrm{~W} /\left(\mathrm{m}^{2} \cdot \mathrm{K}\right)$, and on the side of external environment: $T_{e}=0^{\circ} \mathrm{C}$ and $h_{e}=25 \mathrm{~W} /\left(\mathrm{m}^{2} \cdot \mathrm{K}\right)$. The Physibel Bisco computer program was used for the calculations of heat flux through the window profile, [6]. Spatial discretization was done by triangular uniform mesh with a dimension of $0.5 \mathrm{~mm}$.

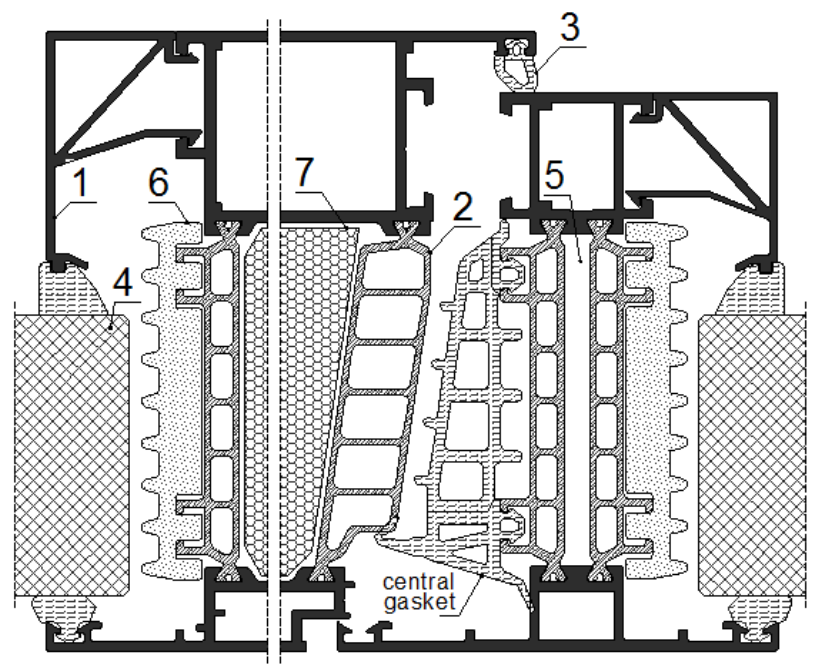

Fig. 2. Elements of window profiles in 'full' thermal variant using the example of profile No. 7: 1) aluminium profile, 2) polyamide thermal break, 3) EPDM gasket, 4) panel (glass unit), 5) narrow air cavity, 6) insulation of the glass unit cavity, 7) insulation of the thermal break cavity.

In order to assess the impact of the method used and the simplifications in defining the emissivity of the surfaces, the thermal transmittance of the profile, $U_{f}$, was determined for the following cases:

- Model 1 (reference) - radiosity method (accurate method), all unpainted surfaces have emissivity $\varepsilon=$ 0.3 , the emissivity of the remaining surfaces is $\varepsilon=0.9$. The model is closest to real situation,

- Model 2 - the single equivalent conductivity method (simplified method) all surfaces (painted and unpainted) have emissivity $\varepsilon=0.9$,

- Model 3 - the single equivalent conductivity method (simplified method), emissivity of unpainted surfaces perpendicular to the direction of heat flow are $\varepsilon_{1}=\varepsilon_{2}$ $=0.3$ (see equation (5)),

- Model 4 - radiosity method, all surfaces (painted and unpainted) have emissivity $\varepsilon=0.9$,
- Model 5 - radiosity method, all surfaces (painted and unpainted) have emissivity $\varepsilon=0.3$,

- Model 6 - radiosity method, unpainted surfaces but only between thermal breaks have emissivity $\varepsilon=0.3$, the emissivity of the remaining surfaces is $\varepsilon=0.9$.

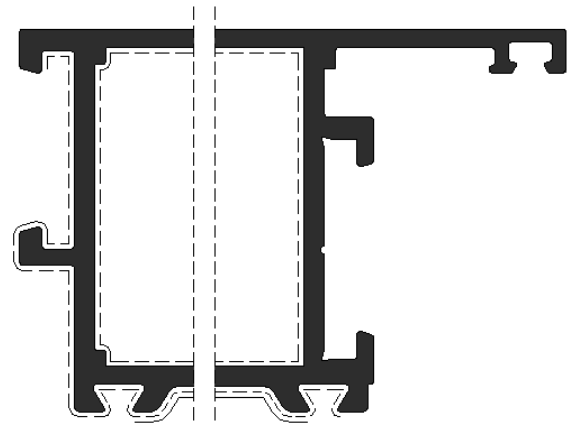

Fig 3. Low-emissivity surfaces in various calculation models: Model 1 - dashed line, Model 3 and 6 - continuous line.

Table 1. Geometrical characteristics of window profiles. Meanings of the heads are as follows: 'wall' - the profile is flank profile to be mounted into the wall, 'fixed' - there is no openable leaf, 'stiff.' - the additional stiffening profile on hot (' $h$ ') or cold ('c') side of the profile, 'insul. (all)' - the number of insulated cavities (all cavities excluding the central gasket) and number of the central gaskets ('cg'), 'corner' - profile for the corner window.

\begin{tabular}{|c|c|c|c|c|c|}
\hline No. & wall & fixed & stiff. & insul.(all) & corner \\
\hline \multicolumn{6}{|c|}{ casement } \\
\hline 1 & & & & $4(5) 1 \mathrm{cg}$ & \\
\hline 2 & $\mathrm{x}$ & & & $3(4) 1 \mathrm{cg}$ & \\
\hline 3 & & & & $5(7) 2 \mathrm{cg}$ & \\
\hline 4 & & & & $4(5) 1 \mathrm{cg}$ & \\
\hline 5 & $\mathrm{x}$ & & & $3(4) 1 \mathrm{cg}$ & \\
\hline 6 & & & & $5(7) 1 \mathrm{cg}$ & \\
\hline 7 & & & & $3(5) 1 \mathrm{cg}$ & \\
\hline 8 & & & & $5(7) 2 \mathrm{cg}$ & \\
\hline 9 & & & & $4(7) 2 \mathrm{cg}$ & \\
\hline 10 & $\mathrm{x}$ & & & $2(4) 1 \mathrm{cg}$ & \\
\hline 11 & & & & $3(5) 1 \mathrm{cg}$ & \\
\hline 12 & & & & $5(7) 1 \mathrm{cg}$ & \\
\hline 13 & $\mathrm{x}$ & & & $2(4) 1 \mathrm{cg}$ & \\
\hline 14 & & & & $4(7) 2 \mathrm{cg}$ & \\
\hline \multicolumn{6}{|c|}{ fixed } \\
\hline 23 & & $\mathrm{x}$ & $\mathrm{c}$ & 3 & \\
\hline 24 & & $\mathrm{x}$ & & 3 & \\
\hline 25 & & $\mathrm{x}$ & $\mathrm{h}$ & 3 & \\
\hline 26 & & $\mathrm{x}$ & $\mathrm{c}, \mathrm{h}$ & 3 & \\
\hline 27 & $\mathrm{x}$ & $\mathrm{x}$ & & 2 & \\
\hline \multicolumn{6}{|c|}{ fixed, with narrow cavities } \\
\hline 18 & & $\mathrm{x}$ & & $2(3)$ & \\
\hline 19 & & $\mathrm{x}$ & $\mathrm{c}$ & $2(3)$ & \\
\hline 20 & & $\mathrm{x}$ & $\mathrm{h}$ & $2(3)$ & \\
\hline 21 & & $\mathrm{x}$ & $\mathrm{c}, \mathrm{h}$ & $2(3)$ & \\
\hline 22 & $\mathrm{x}$ & $\mathrm{x}$ & & $1(2)$ & \\
\hline \multicolumn{6}{|c|}{ corner } \\
\hline 15 & & $\mathrm{x}$ & & $5(7)$ & $\mathrm{x}$ \\
\hline 16 & & $\mathrm{x}$ & & $6(8)$ & $\mathrm{x}$ \\
\hline 17 & & $\mathrm{x}$ & & $4(7)$ & $\mathrm{x}$ \\
\hline
\end{tabular}




\subsection{Thermal transmittance of profiles}

Thermal transmittances of the window profiles, $U_{f}$, obtained by the reference Model 1 are shown in the Tab. 2. The profiles have been ordered in each group according to increasing $U_{f}$-values for the value of the 'full' variant. Considering the 'full' version, one can observe: (i) the most favourable $U_{f}$-values $(0.774 \div 0.813$ $\left.\mathrm{W} /\left(\mathrm{m}^{2} \mathrm{~K}\right)\right)$ have profiles without a central gasket from 'fixed' group, (ii) in the 'corner' group $U_{f}$-values are related to the external (larger) dimension of the profile, which causes a low value of this coefficient $\left(0.564 \div 0.910 \mathrm{~W} /\left(\mathrm{m}^{2} \cdot \mathrm{K}\right)\right)$, (iii) 'casement' group profiles containing at least one non-insulated central gasket does not have significantly worse $U_{f}$-values comparing to profiles with incomplete insulation ('fixed, with narrow cavities' group), (iv) in the 'casement' group, the best $U_{f}$-values were achieved by profiles where the ratio of the semmed width of the insulation elements, $b_{i}$, to the profile width, $b_{f}$, is the largest, which causes profile No. $3\left(b_{i} / b_{f}=0.54\right)$ has a very favourable $U_{f}$-value even with 2 non-insulated central gaskets; elements with narrow insulation inserts (Nos. $10 \div 14$, with $b_{i} / b_{f}$ respectively $0.44,0.34,0.35,0.30,0.29)$ have the worst properties, (v) in both 'fixed' type groups the properties deteriorate with the increase in the number of stiffeners that increase the surface heat transfer (act as heat sinks); in the analysed thermal variant of the fixed groups, the $U_{f}$-value increase due to stiffeners does not exceed $4 \%$ due to the existence of insulation inserts and thermal breaks.

Table 2. Thermal transmittance of window profiles obtained from the reference Model 1 for three different thermal variants

\begin{tabular}{|c|c|c|c|}
\hline \multirow{2}{*}{ No. } & \multicolumn{3}{|c|}{$\mathrm{U}_{\mathrm{f}}\left[\mathrm{W} /\left(\mathrm{m}^{2} \cdot \mathrm{K}\right)\right]$} \\
\cline { 2 - 4 } & standard & plus & full \\
\hline \multicolumn{4}{|c|}{ casement } \\
\hline 1 & 1.797 & 1.586 & 0.896 \\
\hline 2 & 1.801 & 1.673 & 0.918 \\
\hline 3 & 1.792 & 1.632 & 0.940 \\
\hline 4 & 1.732 & 1.478 & 0.948 \\
\hline 5 & 1.724 & 1.563 & 0.985 \\
\hline 6 & 1.777 & 1.577 & 0.989 \\
\hline 7 & 1.702 & 1.418 & 1.022 \\
\hline 8 & 1.692 & 1.481 & 1.031 \\
\hline 9 & 1.749 & 1.559 & 1.032 \\
\hline 10 & 1.719 & 1.550 & 1.070 \\
\hline 11 & 1.597 & 1.245 & 1.111 \\
\hline 12 & 1.637 & 1.360 & 1.116 \\
\hline 13 & 1.551 & 1.324 & 1.145 \\
\hline 14 & 1.594 & 1.332 & 1.145 \\
\hline \multicolumn{4}{|c|}{ fixed } \\
\hline 15 & 1.801 & 1.443 & 0.774 \\
\hline 16 & 1.770 & 1.429 & 0.784 \\
\hline 17 & 1.828 & 1.467 & 0.799 \\
\hline 18 & 1.884 & 1.501 & 0.804 \\
\hline 19 & 1.820 & 1.617 & 0.813 \\
\hline \multicolumn{4}{|c|}{ fixed, with narrow cavities } \\
\hline 20 & 1.561 & 0.989 & 0.989 \\
\hline 21 & 1.603 & 1.000 & 1.000 \\
\hline 22 & 1.614 & 1.010 & 1.010 \\
\hline 23 & 1.660 & 1.027 & 1.027 \\
\hline 24 & 1.556 & 1.152 & 1.111 \\
\hline \multicolumn{4}{|c|}{} \\
\hline
\end{tabular}

\begin{tabular}{|l|l|l|l|}
\hline \multicolumn{4}{|c|}{ corner } \\
\hline 25 & 1.041 & 0.941 & 0.564 \\
\hline 26 & 1.451 & 1.329 & 0.717 \\
\hline 27 & 1.326 & 1.104 & 0.910 \\
\hline
\end{tabular}

In variants with incomplete insulation, i.e. 'plus' and 'standard', the thermal transmittance of profiles, which in the 'full' version in the 'casement' group had good thermal properties, are now among the worst. This is mainly due to the fact that heat exchange in wide voids after removing the insulation is more intense than in narrow voids of non-fully-insulated profiles. In 'fixed' type groups, the properties deteriorate as the number of stiffeners increases $\left(U_{f}\right.$-values increase is up to $7 \%$ and is slightly higher than in the 'full' version). In the 'corner' group, the rules in changing $U_{f}$-values are the same as in the 'casement' group.

Table 3. Range, mean and median of change of $U_{f}$-value with regard to reference Model 1

\begin{tabular}{|c|c|c|c|c|}
\hline $\begin{array}{c}\text { Model } 2 \\
{[\%]}\end{array}$ & $\begin{array}{c}\text { Model } 3 \\
{[\%]}\end{array}$ & $\begin{array}{c}\text { Model } 4 \\
{[\%]}\end{array}$ & $\begin{array}{c}\text { Model } 5 \\
{[\%]}\end{array}$ & $\begin{array}{c}\text { Model } 6 \\
{[\%]}\end{array}$ \\
\hline \multicolumn{5}{|c|}{ Standard } \\
\hline \multicolumn{5}{|c|}{ casement } \\
\hline $10.9 \div 16.1$ & $-7.6 \div 5.3$ & $2.6 \div 17.5$ & $-2.2 \div-0.8$ & $0.4 \div 1.3$ \\
\hline $13.6 / 13.8$ & $-1.5 /-2.6$ & $10.7 / 12.3$ & $-1.3 /-1.3$ & $0.8 / 0.7$ \\
\hline \multicolumn{5}{|c|}{ fixed } \\
\hline $17.4 \div 18.6$ & $-7.4 \div-3.8$ & $15.6 \div 18.6$ & $-2.8 \div-1.5$ & $-0.1 \div 0.1$ \\
\hline $18.0 / 18.0$ & $-4.6 /-4.0$ & $16.6 / 16.2$ & $-2.5 /-2.8$ & $0.0 / 0.1$ \\
\hline \multicolumn{5}{|c|}{ fixed, with narrow cavities } \\
\hline $10.8 \div 11.5$ & $5.9 \div 7.8$ & $0.4 \div 1.0$ & $-5.0 \div-3.2$ & $-0.1 \div 0.3$ \\
\hline $11.1 / 11.2$ & $7.3 / 7.6$ & $0.6 / 0.5$ & $-4.5 /-4.8$ & $0.1 / 0.0$ \\
\hline \multicolumn{5}{|c|}{ Corner } \\
\hline $5.5 \div 19.8$ & $-18.4 \div 2.3$ & $2.3 \div 15.1$ & $-5.3 \div-1.3$ & $1.0 \div 2.9$ \\
\hline $13.5 / 15.2$ & $-5.9 /-1.6$ & $8.3 / 7.4$ & $-3.0 /-2.4$ & $1.7 / 1.2$ \\
\hline \multicolumn{5}{|c|}{ Plus } \\
\hline \multicolumn{5}{|c|}{ casement } \\
\hline$-0.2 \div 13.4$ & $-14.1 \div-6.5$ & $2.7 \div 19.7$ & $-1.4 \div-0.4$ & $0.1 \div 0.4$ \\
\hline $8.1 / 8.9$ & $-10.9 /-11.5$ & $12.1 / 14.1$ & $-0.8 /-0.8$ & $0.2 / 0.1$ \\
\hline \multicolumn{5}{|c|}{ fixed } \\
\hline $15.3 \div 17.0$ & $-16.1 \div-14.9$ & $21.7 \div 22.9$ & $-0.9 \div-0.4$ & $-0.1 \div 0.1$ \\
\hline $16.0 / 15.8$ & $-15.7 /-15.9$ & $22.3 / 22.5$ & $-0.7 /-0.8$ & $0.0 / 0.0$ \\
\hline \multicolumn{5}{|c|}{ fixed, with narrow cavities } \\
\hline$-1.9 \div 2.1$ & $-8.9 \div-6.2$ & $0.6 \div 1.2$ & $-2.0 \div-1.0$ & $0.0 \div 0.2$ \\
\hline$-1.0 \div-1.8$ & $-8.1 /-8.6$ & $0.9 / 0.8$ & $-1.6 /-1.6$ & $0.1 / 0.1$ \\
\hline \multicolumn{5}{|c|}{ Corner } \\
\hline $0.8 \div 18.3$ & $-22.3 \div-3.6$ & $2.9 \div 17.8$ & $-5.1 \div-0.4$ & $1.1 \div 3.7$ \\
\hline $11.1 / 14.3$ & $-11.1 /-7.4$ & 9.9/8.9 & $-2.1 /-0.7$ & 2.0/1.4 \\
\hline \multicolumn{5}{|c|}{ Full } \\
\hline \multicolumn{5}{|c|}{ casement } \\
\hline$-1.3 \div 1.2$ & $-4.6 \div-2.8$ & $0.3 \div 0.9$ & $-1.7 \div-0.7$ & $-0.1 \div 0.5$ \\
\hline$-0.2 / 0.0$ & $-3.6 /-3.5$ & $0.6 / 0.5$ & $-1.3 /-1.4$ & $0.2 / 0.2$ \\
\hline \multicolumn{5}{|c|}{ fixed } \\
\hline$-4.4 \div-2.1$ & $-7.5 \div-6.2$ & $0.0 \div 0.6$ & $-1.8 \div-1.1$ & $-0.3 \div 0.1$ \\
\hline$-3.3 /-3.2$ & $-6.7 /-6.6$ & $0.3 / 0.4$ & $-1.5 /-1.6$ & $-0.1 / 0.0$ \\
\hline \multicolumn{5}{|c|}{ fixed, with narrow cavities } \\
\hline$-1.9 \div 1.7$ & $-8.9 \div-6.9$ & $0.6 \div 1.4$ & $-2.0 \div-1.0$ & $0.0 \div 0.2$ \\
\hline$-1.0 /-1.8$ & $-8.3 /-8.6$ & $1.0 / 1.0$ & $-1.6 /-1.6$ & $0.1 / 0.1$ \\
\hline \multicolumn{5}{|c|}{ Corner } \\
\hline$-4.6 \div-0.4$ & $-11.9 \div-5.2$ & $0.7 \div 1.2$ & $-0.9 \div-0.7$ & $0.3 \div 0.4$ \\
\hline$-1.9 /-0.7$ & $-9.1 /-10.2$ & $0.9 / 0.8$ & $-0.7 /-0.7$ & $0.3 / 0.3$ \\
\hline
\end{tabular}




\subsection{Impact of the type of standard method}

The thermal transmittances of window profiles obtained by EN ISO 10077-2:2017 standard methods marked in this paper by Model 1 and Model 2 and 3 are being compared below. The percent error of determining the $U_{f}$-values was determined according to the formula, eg. [5]:

$$
d U \%=\left(\frac{U_{f, \text { simplified model }}}{U_{f, \text { reference model }}}-1\right) \cdot 100 \%,
$$

where $U_{f}$, simplified model and $U_{f}$, reference model in this paragraph means the thermal transmittance according to the simplified method (single equivalent thermal conductivity method, Model 2 and 3) and the thermal transmittance according to the accurate method (radiosity method, Model 1). According to the formula (6), a negative value of $d U \%$ means an underestimation of the $U_{f}$-value by the simplified model, i.e. a value obtained from the simplified model is more favourable, a positive value - an overestimation of the $U_{f}$-value, i.e. a less favourable value is obtained from the simplified model. Fig. $4 \div 6$ shows $d U \%$ values for all profiles. In Tab. 3 the range, average and median of the $d U \%$ values for individual thermal variants and profile groups are shown.

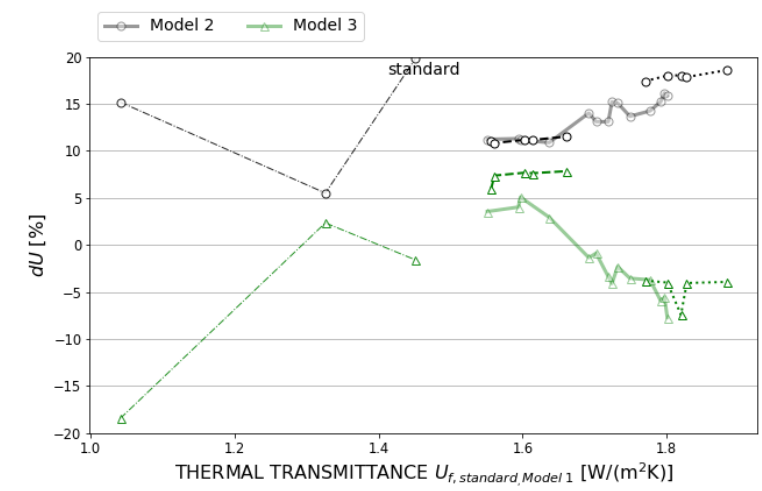

Fig. 4. Percent error of different models of the simplified method for the 'standard' variant of the profiles (noninsulated); line styles for the groups of windows are as follows: solid - casement window, dotted - fixed, all cavities insulable, dashed - fixed, with narrow non-insulable cavities, dash-dotted - corner profiles.

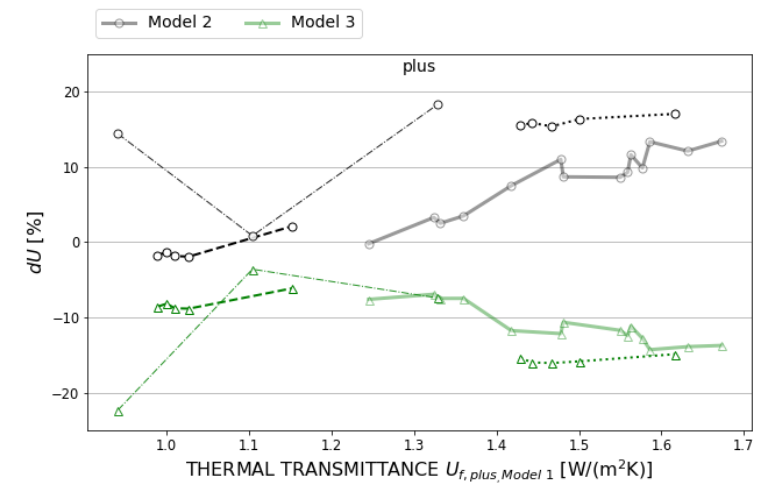

Fig. 5. Percent error of different models of the simplified method for the 'plus' variant of the profiles (IGU cavities insulated); the line styles are as on Fig. 4.

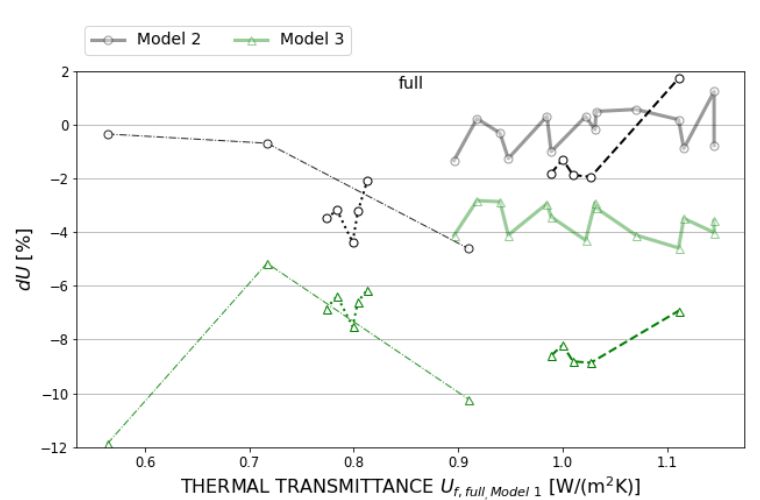

Fig. 6. Percent error of different models of the simplified method for the 'full' variant of the profiles (IGU and thermal break cavities insulated); the line styles are as on Fig. 4.

$U_{f}$-values obtained from the simplified method with low emissivities (Model 3, this model is often used in practice) for the 'full' and 'plus' variants are more favourable than that from the accurate Model 1. In the case of the 'full' thermal variant, the values are $3 \div 9 \%$ lower, in the case of the 'plus' variant - by $6 \div 16 \%$, Tab. 3 . The given values do not include profiles from the 'corner' group, for which the differences reach even $22 \%$. This is probably due to a simplified way of creating an equivalent rectangular section (see sections 2 and 4). In the 'standard' variant, profiles with wide air voids have underestimated $U_{f}$-values, profiles with narrow voids - overestimated, Fig. 4. (solid, dashed and dotted lines with round markers). Considering the most numerous group with the most diverse profiles ('casement' group), it can be observed that the magnitude of $d U \%$ is roughly proportional to the $U_{f^{-}}$ value according to Model 1, Fig. 4 and 5 (thick line with round markers), or slightly fluctuates around a constant value, Fig. 6.

Simplified method without surfaces of lowemissivity (Model 2) overestimated $U_{f}$-values up to $20 \%$ for 'standard' and 'plus' thermal variant and up to $5 \%$ for 'full' version.

\subsection{Impact of the simplifications when modelling low-emissivity surfaces}

The impact of varying degrees of accuracy in modelling low-emissivity surfaces was determined according to the formula (6) where the results obtained from Model 4, 5 and 6 were substituted for the $U_{f}$, simplified model. In general, the impact of individual simplifications is as could be expected: Models 4 and 6 with more surfaces of unfavourable emissivity $\varepsilon=0.9$ than in Model 1 overestimated $U_{f}$-values, Model 5 with all surfaces of low-emissivity $\varepsilon=0.3$ - underestimated the $U_{f}$-values, Tab. 3, Fig. $7 \div 9$. The range of error varies significantly depending on the type of simplification and the thermal variant of profile. For the 'full' variant and radiosity method, the deviation from the reference model did not exceed $2 \%$ regardless of the modelling technique. The 
smallest deviation (underestimation) of the $U_{f}$-values were obtained for Model 6 and it was between 0.5 and $-0.3 \%$. For the 'plus' variant, setting high emissivity for all surfaces resulted in an overvaluation of $U_{f}$-value by $0.6-22.9 \%$. The highest values occurred in the 'fixed' group (dotted line with circle marks on Fig. 8), the smallest $(0.6-1.2 \%)$ in the group 'fixed, with narrow cavities' (dashed line with circle marks on Fig. 8) and in those profiles from the 'casement' group, which have a large number of narrow air cavities. The results from Model 5 do not exceed the values from the reference model by more than $2 \%$ for normal profiles and $5 \%$ for profiles from the 'corner' group. The smallest deviations were again obtained for Model 6. Based on the observation of the course of values in the most diverse 'casement' group, it can be said that the prediction error on the basis of Model 3 becomes greater with the increase in the $U_{f}$-value, whereas the predictions on the basis of Model 5 and 6 have constant error, Fig. 8. (solid lines). Similar conclusions as for 'plus' variant could be drawn for the 'standard' thermal variant. Model 5 and 6 errors are slightly larger in 'standard' variant and are in the ranges of $-5 \div-0.8 \%$ and $0.1 \div 1.3 \%$, respectively.

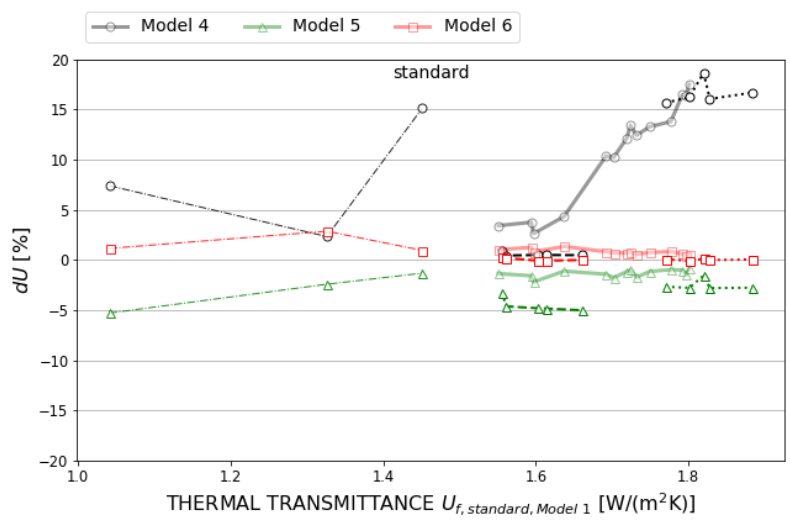

Fig. 7. Percent error of different models of the radiosity method for the standard variant of the profiles (non-insulated); the line styles are as on Fig. 4

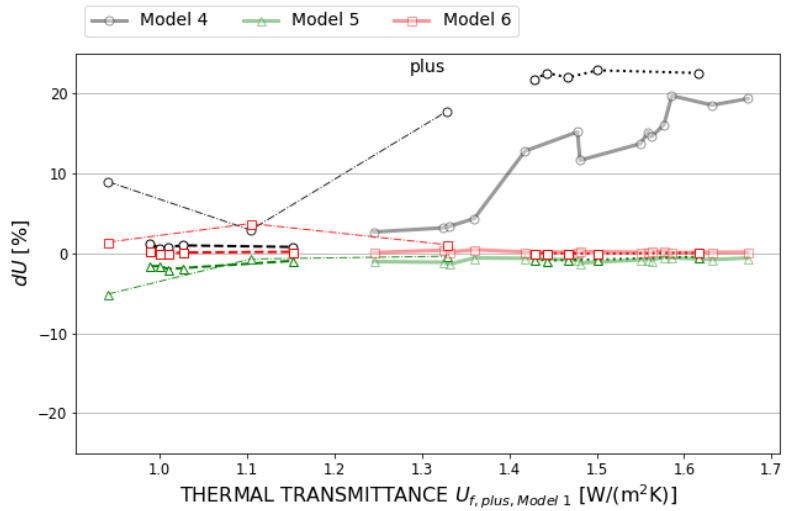

Fig. 8. Percent error of different models of the radiosity method for the plus variant of the profiles; the line styles are as on Fig. 4

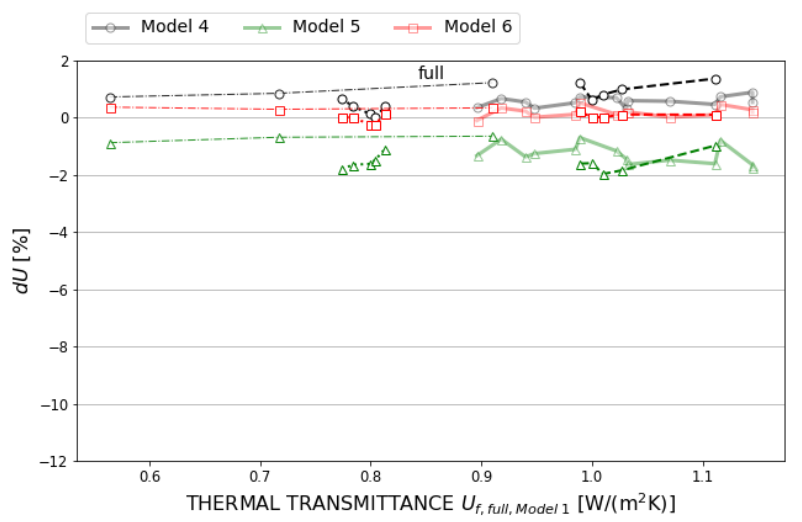

Fig. 9. Percent error of different models of the radiosity method for the full variant of the profiles; the line styles are as on Fig. 4

\section{Remarks on modelling radiation}

The main source of differences between the two standard methods is the approach to the radiation heat exchange. In order to assess the magnitude of these differences, a series of calculations was made for a single cell of the window profile. Material and geometric characteristics of the cell are presented in Fig. 10 and Tab. 4. The results form ray tracing type method were compared to the results of equivalent conductivity method. In the second case, radiation exchange is modelled by introducing a solid material in the place of the air with a heat transfer coefficient defined as:

$$
\lambda_{e q, r}=d \cdot h_{r},
$$

where $d$ is depth of the air cavity of the cell, $h_{r}$ was calculated according to the formula (5).
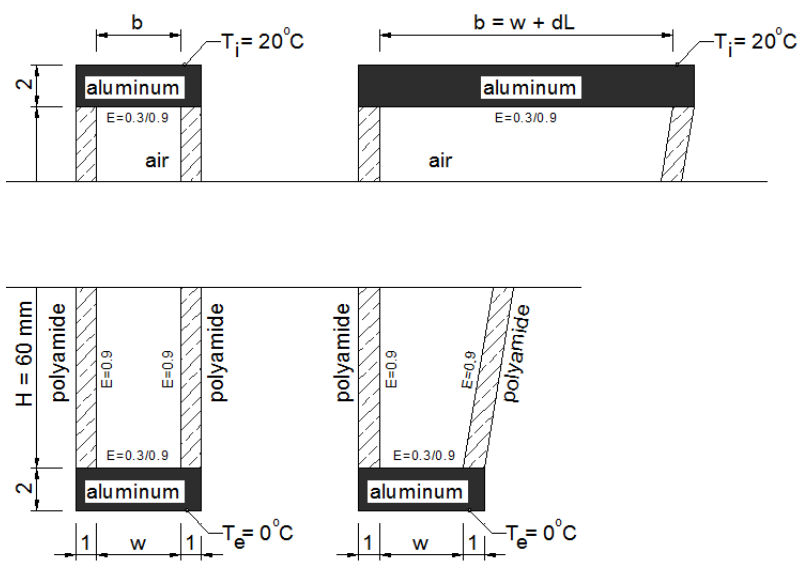

Fig. 10. Rectangular and trapezoidal cell used to asses accuracy of equivalent conductivity due to radiation heat exchange.

In the case of a trapezoidal void, all characteristics relate to the equivalent rectangular section (see Section 2 ). The surface emissivity of polyamide was always 0.9 , aluminium -0.9 or 0.3 . Heat flow in the air gap as a result of conduction and convection was not included. For comparison, the heat flow through the solid elements 
of the cells obtained from the model without any radiation exchange is given. Boundary conditions of the Dirichlet type were used for the analysis. Difference between the length of upper and lower side of trapezoidal cell ( $d L$ on Fig. 10) was set $10 \mathrm{~mm}$.

Table 4. Characteristics of cavities $(b$ - width $[\mathrm{mm}], d / b$ height/width ratio [-], $\lambda_{e q, r}-$ equivalent thermal conductivity of air due to radiation heat exchange for emissivites of aluminium 0.9 and 0.3

\begin{tabular}{|c|c|c|c|c|c|c|}
\hline Quantity & \multicolumn{7}{|c|}{ rectangular } \\
\hline \multicolumn{8}{|c|}{ trapezoidal } \\
\hline$b$ & 4 & 5 & 10 & 20 & 40 & 60 \\
\hline$d / b$ & 15 & 12 & 6 & 3 & 1.5 & 1 \\
\hline$\lambda_{\text {eq, } r 09}$ & 0.131 & 0.132 & 0.137 & 0.147 & 0.165 & 0.179 \\
\hline$\lambda_{\text {eq, } r 03}$ & 0.028 & 0.028 & 0.030 & 0.032 & 0.036 & 0.039 \\
\hline \multicolumn{7}{|c|}{} \\
\hline$b$ & 14 & 15 & 20 & 30 & 50 & 70 \\
\hline$d / b$ & 4.29 & 4.00 & 3.00 & 2.00 & 1.20 & 0.86 \\
\hline$\lambda_{\text {eq,r } 09}$ & 0.091 & 0.095 & 0.110 & 0.130 & 0.155 & 0.171 \\
\hline$\lambda_{\text {eq, } r 03}$ & 0.020 & 0.020 & 0.024 & 0.028 & 0.033 & 0.037 \\
\hline
\end{tabular}

From Figs. 11 and 12 results that the increase in the heat flux caused by the radiation heat exchange is significant. In the case of aluminium emissivity $\varepsilon_{A l}=$ 0.9 total heat flux is 1.4 to 20.5 times higher than the conductive heat flux through the polyamide and aluminium elements in the case of a rectangular section and 2.3 to 22.6 times higher in the case of the trapezoidal section depending on the $d / b$ ratio. Lower values of aluminium emissivity lead to a decrease in heat exchange intensity, and the flux in this case increases for rectangular and trapezoidal cross sections respectively by $1.3 \div 8$ times and $2.4 \div 8.7$ times comparing to the model without radiation. The general rule is that the wider the air gap, the greater the contribution of radiation exchange.

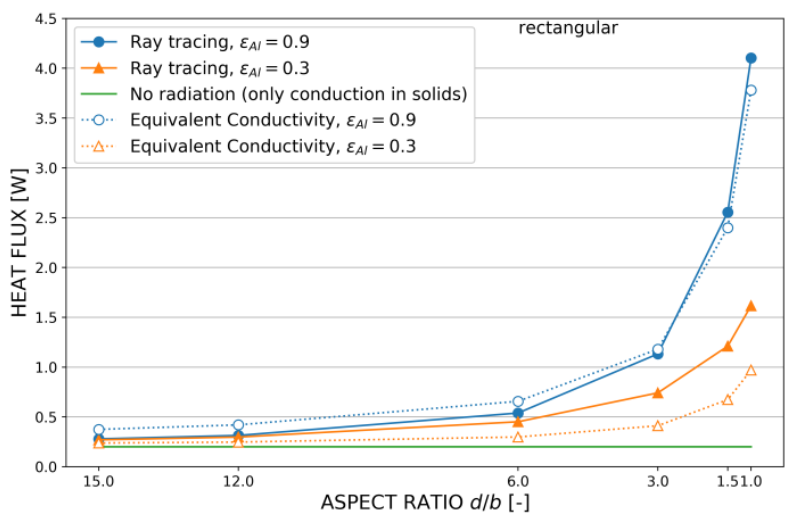

Fig. 11. Heat flux through the rectangular cell for the emissivity of the aluminium equal to 0.9 or 0.3 by the ray tracing method and equivalent conductivity method.

The percentage error of determining the heat flux by the method of equivalent thermal conductivity in the case of $\varepsilon_{A l}=0.9$ and a rectangular cross-section varies from $-25.5 \%$ to $8.6 \%$ for decreasing $d / b$ values (therefore for narrower voids the heat flow is significantly overestimated), for the trapezoidal section varies from $1.9 \%$ to $15.1 \%$ (the flux is underestimated). Assuming lower values of aluminum emissivity $\varepsilon_{A l}=$ 0.3 , the error of determining the heat flux is $13.6 \div 66.6 \%$ and $59.2 \div 88.34 \%$ for rectangular and trapezoidal crosssections respectively. Thus, the method of equivalent heat transfer coefficient for radiation exchange leads to a large underestimation of heat flow through the window profile cells. Assuming that the emissivity of aluminium is 0.9 instead of 0.3 , for narrower air voids $(d / b \geq 6$ for the rectangular cavity and $d / b \geq 3$ for the trapezoidal cavity) more accurate results will be obtained, and for wider air cavities the results will be overestimated, and therefore more conservative energy demand will be obtained for the building.

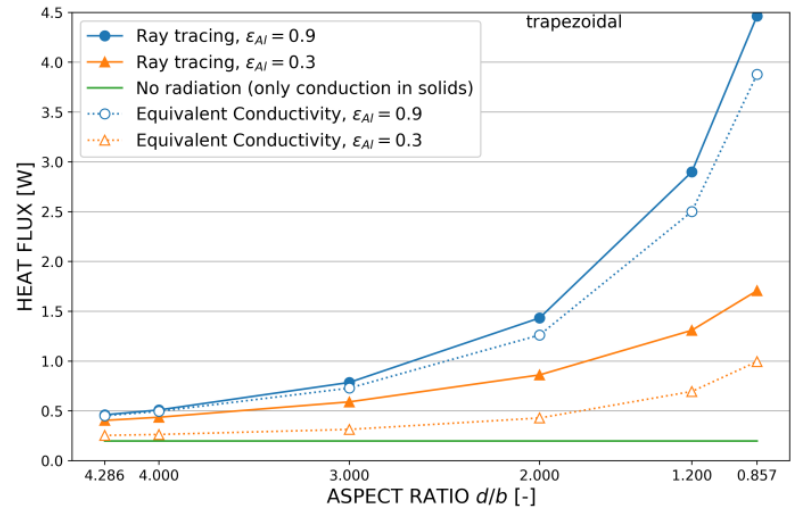

Fig. 12. Heat flux through the trapezoidal cell for the emissivity of the aluminium equal to 0.9 or 0.3 by the ray tracing method and equivalent conductivity method.

\section{Conclusions}

On the basis of the presented results obtained for a given series of diversified products it can be stated what follows:

- considering cavities in the area of thermal brakes: in profiles with insulation inserts it is worth to create larger cavities, in profiles without insulation inserts the narrower cavities,

- the single equivalent thermal conductivity method (simplified method) of the EN ISO 10077-2:2017 standard generally overestimate (models without low-emissivity surfaces) or underestimate (models with low emissivity surfaces) the thermal transmittance of profiles up to $19 \%$ comparing to values from the radiosity method,

- not including low-emissivity surfaces in the model with radiosity method can cause underestimation of up to $22 \%$,

- in terms of the magnitude of absolute value of prediction error: assuming all surfaces as lowemissive is better choice than assuming all surfaces as painted,

- the better insulated profile (the major of the heat flow is through the conduction in the solids) the better is the accuracy of simplified models and methods; from models with simplified method better prediction for well insulated profiles gives model without including low-emissivity surfaces. 
Concluding, on the basis of the results of our work it can be strongly recommended to use radiosity method. The economically reasonable use of simplification when low-emissivity surfaces are present only between thermal breaks gives very good accuracy in predicting thermal transmittance of aluminium profiles.

\section{References}

1. EN ISO 10077-2:2017 Thermal performance of windows, doors and shutters - Calculation of thermal transmittance — Part 2: Numerical method for frames

2. F. Asdrubali, G. Baldinelli, F. Bianchi, Energ. Buildings, 60, 298 (2013)

3. P. Noyé, J.B. Laustsen, S. Svendsen, Nordic J. B. Phys. 3, 1 (2004)

4. A. Gustavsen, Ch. Kohler, D. Arasteh, D. Curcija, ASHRAE Transactions, 111, 587 (2005)

5. https://en.wikipedia.org/wiki/Relative change and difference

6. BISCO computer program to calculate twodimensional steady state heat transfer in free-form objects version 11.0w, PHYSIBEL, (2016) 